\title{
Minimization of Consumption of Energy using Clustering and Fault Tolerance using Recovery Node in Mobile Wireless Sensor Networks
}

\author{
Ankita Chaudhry ${ }^{1}$, Nivit Grewal ${ }^{2}$ \\ Scholar ${ }^{1}$, Assistant Professor ${ }^{2}$
}

\begin{abstract}
Mobile Wireless Sensor Network (MWSN) is a latest emerging technology during last decades, the researchers are doing efforts to find a low cost and low power way out for mobile wireless sensor network. Many clustering based algorithms are available in mobile wireless sensor networks. The proposed study focuses on application of a distributed fault-tolerant clustering algorithm using LEACH in mobile wireless sensor networks. The fault has been removed by using back up cluster or by setting the root information as a prefix. The simulated results shows that LEACH routing protocol is better than HEED routing protocol in terms of energy consumption of mobile sensor nodes and extensively improve the network lifetime.
\end{abstract}

\section{Keywords}

$\mathrm{CH}$, Clustering, Fault Tolerance, Mobility, MWSN, WSN

\section{INTRODUCTION}

WSN can connect information world and physical world together[1]. Nowadays, WSN is being used in a variety of applications such as battlefield surveillance, monitoring of land, remote healthcare and monitoring of environment. Several challenges occurs while designing algorithms and protocols of WSNs like connectivity needs to be maintained and network lifetime needs to be maximized. Mobile or remote devices are the best approach to resolve the WSNs problems in an efficient manner. MWSN is a specific category of WSN where mobility acts as a primary part in the execution of application[2]. In recent years, researchers are entirely concentrated to maintain mobility in WSN.

Mobile wireless sensor network (MWSNs) can essentially be characterized as a remote sensor system (WSN) in which the sensor hubs are mobile[3]. MWSNs are a littler, developing field of exploration rather than their settled ancestor. MWSNs are substantially more flexible than static sensor organizes as they can be sent in any situation and adapt to fast topology changes[4]. Nonetheless, huge numbers of their applications are comparative, for example, environment observing or reconnaissance. Commonly the hubs comprise of a radio handset and a microcontroller fueled by a battery. And also a sensor for recognizing light, warm, mugginess, temperature, and so on [1]. The clustering plays a vital role in energy saving with better resource allocation[5].

For a basic good performance in mobile wireless sensor network, clustering of large multi-hop wireless network is really essential. In MWSN the clustering is divided into two basic parts:- a) Periodic selection of cluster head.

b) Assignment of each node to single node or multiple nodes.

MWSN contains large number of tiny nodes distributed over large network with one or more powerful sinks. All nodes have limited supply and have the advantage of handling information sensing, data processing etc. Resources are constraint over power supply, processing capability and transmission bandwidth. Based upon network band width, routing protocols are divided into two categories:-

(i) Flat routing

(ii) Hierarchical routing

(i) Flat routing- All nodes have same task and same functionality in network. For small scale networks flat routing is better than other. For large network it is not feasible because it uses limited resources, but they consume more data processing and network usage.

(ii) Hierarchical routing- In this routing nodes perform different task in MWSN and are organized into many clusters according to requirement. Each cluster is made up of a head denoted as a cluster head $(\mathrm{CH})$ and other sensor nodes, and the $\mathrm{CHs}$ can be devised into further hierarchical levels. Nodes with maximum energy act as $\mathrm{CH}$ and perform the chore of processing data and transmission of information, while nodes with less energy act as MNs and execute the task of information sensing.

\subsection{Various Clustering Parameters}

Some important parameters with regard to the whole clustering procedure in MWSNs [6]are:

Number of clusters (cluster count):In latest probabilistic and randomized grouping calculations the $\mathrm{CH}$ race and arrangement methodology lead regularly to variable number of groups. The set of $\mathrm{CHs}$ are foreordained and hence the quantity of groups is preset. The quantity of bunches is typically a basic parameter with respect to the effectiveness of the aggregate steering convention.

Nodes and CH mobility: The stationary sensor nodes and stationary $\mathrm{CHs}$ led to stable clusters with facilitated intracluster and intercluster network management. In contrast, if the $\mathrm{CHs}$ or the sensor nodes themselves are mobile, the cluster membership for each sensor node will change dynamically.

Nodes types and roles: In homogeneous environments, all the sensor nodes have the same capacity and out of the deployed sensor nodes is designated as $\mathrm{CHs}$ but the $\mathrm{CHs}$ are assumed to be furnished with specifically higher computation 
and communication resources than other in heterogeneous environments.

Cluster formation methodology: In latest methodologies, when $\mathrm{CHs}$ are simply customary sensors hubs and time effectiveness is an essential outline measure, grouping is being performed in a conveyed way without coordination. In few prior methodologies a concentrated (or half and half) approach es taken after; one or more organizer hubs are utilized to segment the entire system disconnected from the net and control the bunch enrollment.

Cluster-head selection: The cluster heads $(\mathrm{CHs})$ in some proposed algorithm(i.e., in heterogeneous environments) can be pre-assigned. In most cases however (i.e., in homogeneous environments), the $\mathrm{CHs}$ are selected from the number of nodes deployed using some particular criteria i.e. on the basis of energy or connectivity.

\begin{abstract}
Algorithm complexity: In latest calculations the quick end of the executed convention is one of the essential configuration objectives. Accordingly, the time many-sided quality or merging rate of most bunch development methods proposed these days is steady (or simply subject to the quantity of $\mathrm{CHs}$ or the quantity of bounces). In some prior conventions, be that as it may, the multifaceted nature time has been permitted to rely on upon the aggregate number of sensors in the system.
\end{abstract}

Multiple levels: In a few distributed methodologies the idea of a multi-level bunch progression is acquainted with accomplish stunningly better vitality dispersion and aggregate vitality utilization (as opposed to utilizing stand out group level). The changes offered by multi-level grouping are to be further considered, particularly when we have huge systems and between $\mathrm{CH}$ correspondence proficiency is of high significance.

Overlapping: The node overlapping within different clusters can be for the efficient routing or for faster cluster execution. Many of the routing protocols, however, still try to minimize the rate of overlapping or do not support the concept of overlapping at all.

\subsection{Various Clustering Techniques}

\subsubsection{Leach Protocol}

LEACH (Low Power Adaptive Clustering Hierarchy) is one of the energy efficient hierarchical clustering algorithm for the sensor networks proposed for less power consumption. In $\mathrm{LEACH}$, the clustering task is rotated among all the mobile nodes based on duration. Each cluster head forwards the data or information to the base station (BS)[7]. It is an applicationspecific data dissemination protocol that uses clusters to prolong the life of the sensor network[8].

LEACH is based on an aggregation (or fusion) technique that combines or aggregates the original data into a smaller size of data that carry only meaningful information to all individual sensors. LEACH divides the a network into several cluster of sensors, which are constructed by using localized coordination and control not only to reduce the amount of data that are transmitted to the sink, but also to make routing and data dissemination more scalable and robust. Given that energy dissipation of the sensor depends on the distance and the data size to be transmitted, LEACH attempts to transmit data over short distances and reduce the number of transmission and reception operations[9]. The key features of LEACH are:
Randomized rotation of the $\mathrm{CH}$ and corresponding clusters,

Local compression to reduce global communication,

(iii) Localized coordination and control for cluster set-up and operation.

LEACH uses a randomize rotation of high-energy $\mathrm{CH}$ position rather than selecting in static manner, to give a chance to all sensors to act as $\mathrm{CHs}$ and avoid the battery depletion of an individual sensor and dyeing quickly[10]. The operation of LEACH is divided into rounds, each of which has mainly two phases as given below:

(i) A setup phase to organize the network into clusters, $\mathrm{CH}$ advertisement, and transmission schedule creation.

(ii) A steady-state phase for data aggregation, compression, and transmission to the sink.

\subsubsection{HEED}

An alternate enhanced and exceptionally mainstream vitality effective convention is HEED (Hybrid Energy- Efficient Distributed Clustering). In HEED,the $\mathrm{CH}$ hubs are picked taking into account two essential parameters, remaining vitality and intra bunch correspondence cost. Leftover vitality of every hub is utilized to probabilistically pick the beginning set of CHs. Then again, intra bunch correspondence expense mirrors the hub degree or hub's closeness to the neighbor and is utilized by the hubs as a part of choosing to join a group or not. Subsequently, dissimilar to LEACH, in HEED the $\mathrm{CH}$ hubs are not chosen arbitrarily. Just sensors that have a high leftover vitality are relied upon to end up $\mathrm{CH}$ hubs. Likewise, the likelihood of two hubs inside the transmission scope of one another getting to be CHs is little. Not at all like LEACH, has this implied that $\mathrm{CH}$ hubs are decently disseminated in the system. In addition, when picking a bunch, a hub will correspond with the $\mathrm{CH}$ that yields the least intra group correspondence cost. In HEED, every hub is mapped to precisely one group and can specifically speak with its $\mathrm{CH}$. Additionally; vitality utilization is not thought to be uniform for all the hubs[2].

This paper focuses on application of a distributed faulttolerant clustering algorithm in mobile wireless sensor networks. The research paper has been organized as shown below: section II summarizes the proposed work; section III represents simulation environment; section IV describes the simulation results and finally conclusion is presented in section V.

\section{THE PROPOSED MODEL}

The proposed model focuses on the implementation of LEACH protocol in order to minimize consumption of energy and fault tolerance in mobile wireless sensor networks. Figure 1 depicts the workflow of the proposed system as:-

Creating MWSN Scenario:- First of all mwsn scenario is created by arranging 50 mobile sensor nodes randomly in the sensor fields.

Cluster formation methodology:- In latest methodologies, when $\mathrm{CHs}$ are simply customary sensors hubs and time effectiveness is an essential outline measure, grouping is being performed in a conveyed way without coordination. In few prior methodologies a concentrated (or half and half) approach es taken after; one or more organizer hubs are 
utilized to segment the entire system disconnected from the net and control the bunch enrollment.

Implementation of LEACH protocol :-Leach protocol is installed and cluster formation is done on the basis of LEACH. Node with the highest energy is elected as a cluster Head . Properties of this algorithm include:

- Cluster based

- Random cluster head selection each round with rotation

- Cluster membership adaptive

- Data aggregation at cluster head

- Cluster head communicate directly with sink or user

- Communication done with cluster head via TDMA

- CDMA across clusters

Cluster Head Selection:- Nodes in a cluster Communicate in Hierarchy like cluster head communicate with sub-cluster head \& sub cluster head communicate with cluster member in the end all cluster heads communicate with base station.

Fault Tolerance:- : Some sensor nodes may be hindered because of physical harm, insufficient energy or some other issues. The failure of mobile sensor nodes should not affect the general working of the whole sensor system. In the event that numerous nodes fail, MAC must suit development of new connections and recovery of the data from the base stations by gathering the information from the cluster heads.

Quality of Service Parameters:- In the end parameter analysis is done on the basis of Quality of service parameters (QOS) like Lifetime of nodes, Energy consumption of nodes, Throughput, Loss of Packets.

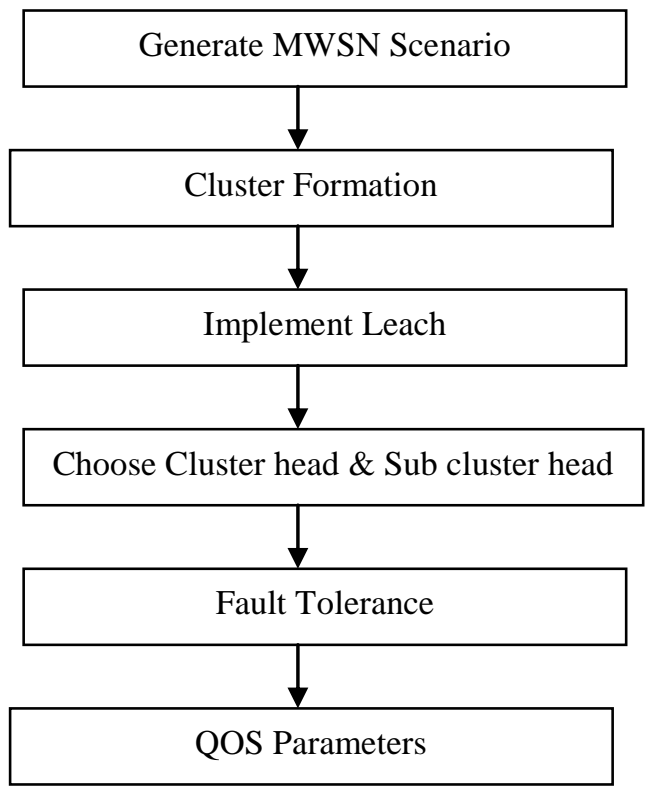

Fig 1. Work Flow of Proposed system

\section{SIMULATION ENVIRONMENT}

The simulation environment has been set up to analyse and evaluate the performance of the proposed work. This paper uses NS2 simulator, developed by UC Berkeley to simulate the work .

\subsection{Simulation Platform}

NS2 simulation tool is an open source discrete event network simulator for research in networking areas[11].It provides a complete development environment for the evaluation of performance of communication networks and distributed systems.

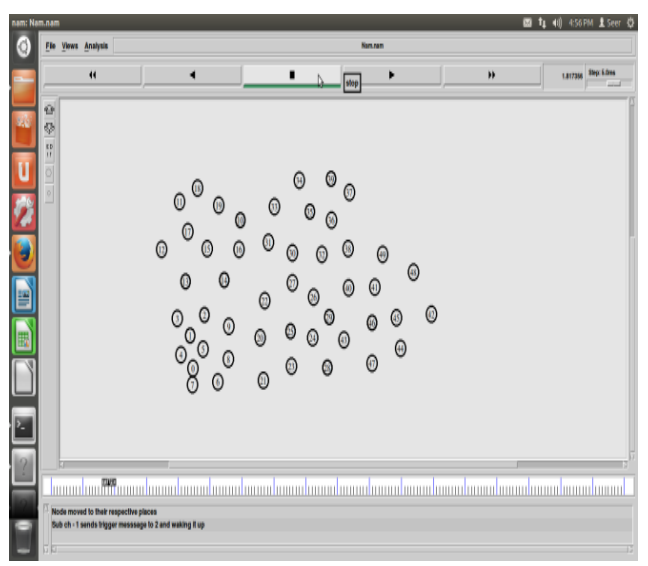

Fig 2: Random deployment of 50 mobile nodes

In above figure initialization of nodes is shown. These nodes are initialized by assigning the appropriate scenario parameter like topology used, antenna type used, protocol used and ground area etc.

\subsection{Simulation Setup}

As shown in figure 2, 50 mobile sensor nodes were arranged randomly in the field $1000 \mathrm{~m}^{*} 1000 \mathrm{~m}$ in the sensor fields. The settings of other simulation parameters used are displayed in table1.

Table 1. Simulation Parameters

\begin{tabular}{|c|c|}
\hline Parameter Name & Parameter Value \\
\hline Propagation Medium & Two way Ground \\
\hline Mac Type & $802.11 \mathrm{p}$ \\
\hline $\begin{array}{c}\text { Number of Mobile } \\
\text { Nodes }\end{array}$ & 50 \\
\hline $\begin{array}{c}\text { Protocol Used } \\
\text { Number of Clusters }\end{array}$ & LEACH \\
\hline Antenna Type used & Omni Antenna \\
\hline Initial Energy of Nodes & 100 J(Per node) \\
\hline Simulation Grid Size & $1000 \mathrm{~m} * 1000 \mathrm{~m}$ \\
\hline Bandwidth & 28.8 kbps \\
\hline Queue Type & Drop tail Priqueue \\
\hline Simulation Time & 20 sec \\
\hline
\end{tabular}




\subsection{Performance Metrics for Evaluation}

The performance metrics are used to analyse and evaluate the performance of the simulation of the proposed work

Simulation Time: The time taken for each simulation to run or it can be said as the time between start and end of it.

Throughput : It is an average rate of successful message delivery over a network. It is measured in bits/sec or packets/sec.

Energy Consumption: It refers to the amount of energy consumed in transferring data from source to destination.

Loss: It refers to the amount of data that is not received to the destination and is dropped from the network. It is expressed in bits or packets .

Lifetime : It is the time at which the first network node runs out of energy to send a packet

\section{SIMULATION RESULTS}

This section represents the comparison of performance of the proposed work with the existing work using parameters such as average energy consumed, alive nodes, packet loss and lifetime of nodes.

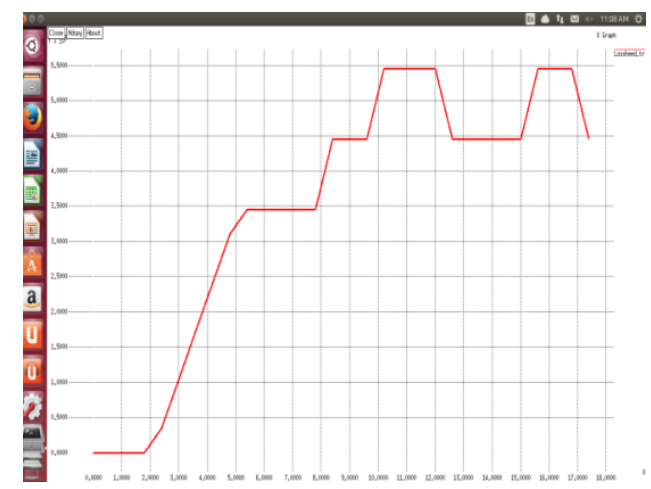

Fig 3(a): Represents Loss of packets using HEED

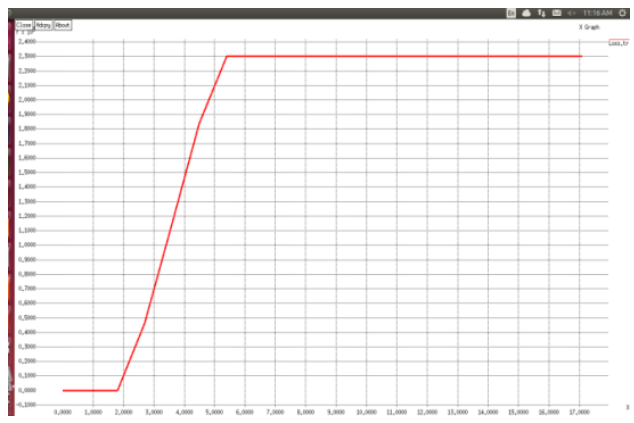

Fig 3(b): Represents Loss of packets using LEACH

From the above graphs fig 3(a) represents loss with HEED protocol. On the other hand fig 3(b) representing the same with LEACH protocol. It has been noticed that the LEACH protocol gives better results because with the LEACH loss is less as compared to HEED protocol.

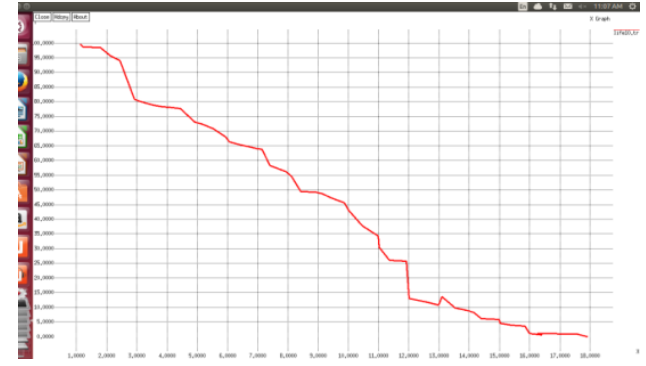

Fig 4(a): Represents Lifetime of node 10 using HEED

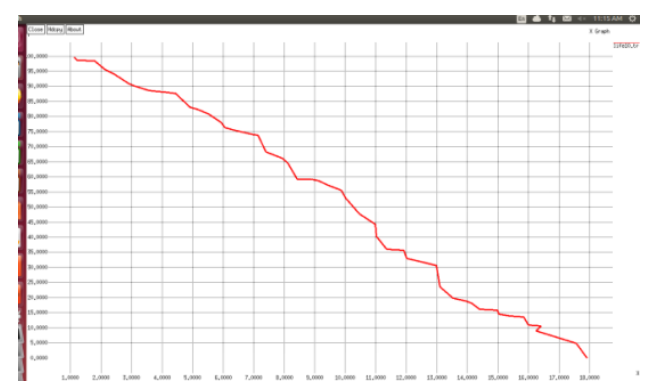

Fig 4(b): Represents Lifetime of node 10 using LEACH

From above figures fig 4(a) represents Lifetime of node 10 with HEED protocol. On the other hand fig 4(b) representing the same with LEACH protocol. It has been noticed that the LEACH protocol gives better results because with LEACH its lifetime becomes 0 in $18^{\text {th }}$ iteration and on the other hand with HEED protocol its lifetime become 0 in $15^{\text {th }}$ iteration.

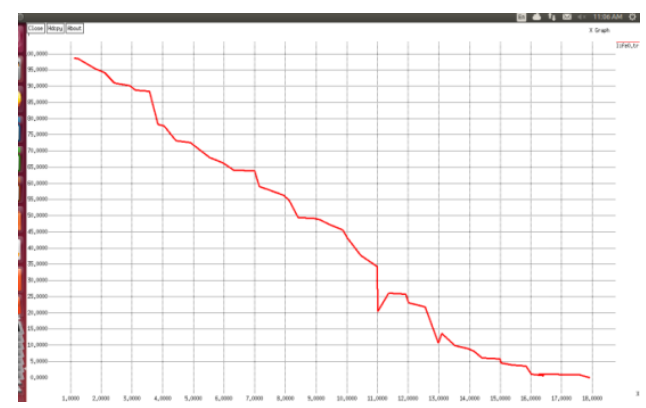

Fig 5(a): Represents Lifetime of node 0 using HEED

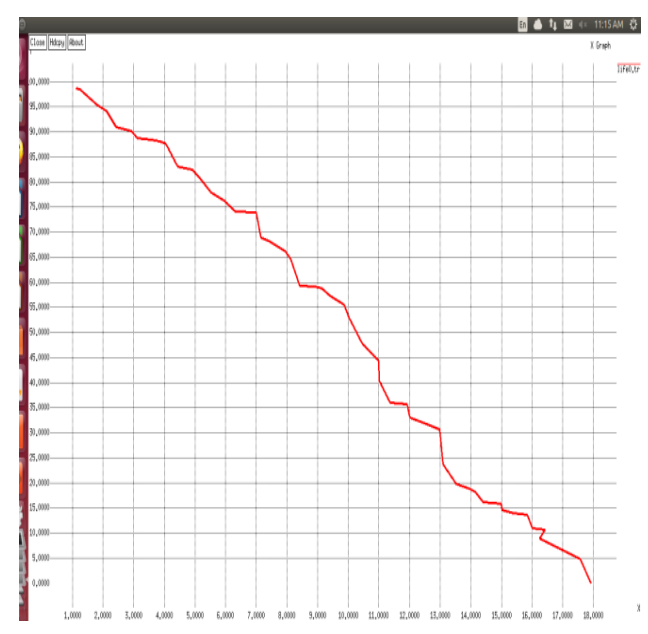

Fig 5(b): Represents Lifetime of node 0 using LEACH 
From above fig 5(a) represents Lifetime of node 0 with HEED protocol. On the other hand fig 5(b) representing the same with LEACH protocol. It has been noticed that LEACH protocol gives better results because with LEACH its lifetime becomes 0 in $18^{\text {th }}$ iteration and on the other hand with HEED protocol its lifetime becomes 0 in $15^{\text {th }}$ iteration.

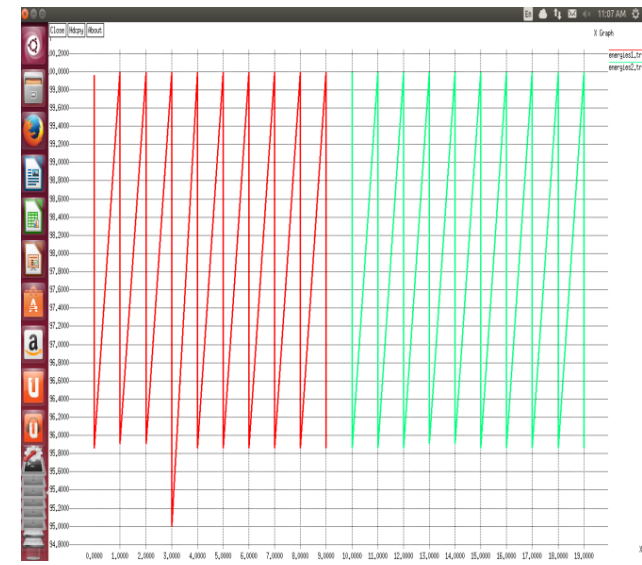

Fig 6(a): Energy consumption of node 1 and node 2 using HEED

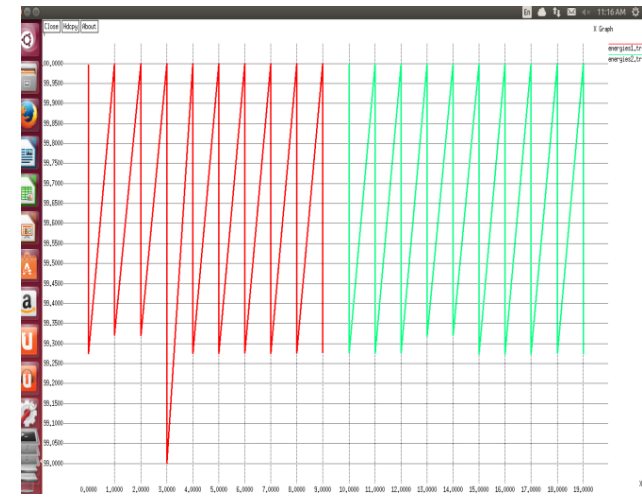

Fig 6(b): Energy consumption of node 1 and node 2 using LEACH

From the above figures fig 6(a) represents energy for node 1 and 2 with HEED protocol. On the other hand fig 6(b) depicting the energy consumption for node 1 and 2 with LEACH protocol. It has been noticed LEACH protocol gives better result because it only consumes very less amount of the energy.

\section{CONCLUSION}

In mobile wireless sensor network, sensor nodes relocate themselves with the intention of covering area to be monitored or to replace uncharged or defective sensor nodes. In some situations, sensors are just dropped from a height or thrown parabolic and supposed to position themselves to track something or monitor an area. In such cases, energy becomes a vital issue for sensor nodes as they do not contain any battery recharging facility as well as they are very small in size hence cannot resist high battery capacity. For such an environment, energy needs to be conserved at each step either sensing or processing or communicating or moving . This paper addresses the considerable comparison in minimization of consumption of energy and fault tolerance in mobile wireless sensor networks using LEACH protocol and HEED routing protocol. According to aforementioned simulated results is the evidence for LEACH routing protocol is better than HEED routing protocol in terms of energy consumption of mobile sensor nodes and extensively improve the network lifetime.

\section{REFERENCES}

[1] J. Rezazadeh, M. Moradi, and A. S. Ismail, "Mobile Wireless Sensor Networks Overview," Int. J. Comput. Commun. Networks, vol. 2, no. 1, pp. 17-22, 2012.

[2] S. A. Munir, B. Ren, W. Jiao, B. Wang, D. Xie, and J. Ma, "Mobile Wireless Sensor Network: Architecture and Enabling Technologies for Ubiquitous Computing," 21st Int. Conf. Adv. Inf. Netw. Appl. Work., pp. 113-120, 2007.

[3] P. Kumari, M. Singh, and P. Kumar, "Cluster head selection in mobile wireless sensor networks: a survey," Intl. Conf. Adv. Comput. Commun., pp. 978-981, 2013.

[4] R. U. Anitha and P. Kamalakkannan, "Energy efficient cluster head selection algorithm in mobile wireless sensor networks," in 2013 International Conference on Computer Communication and Informatics, 2013, pp. 15.

[5] V. V. Deshpande and A. R. Bhagat Patil, "Energy efficient clustering in wireless sensor network using cluster of cluster heads," in 2013 Tenth International Conference on Wireless and Optical Communications Networks (WOCN), 2013, pp. 1-5.

[6] J. Lakhotia and R. Kumar, "CLUSTER BASED ROUTING PROTOCOLS FOR MOBILE WIRELESS SENSOR NETWORK: A REVIEW," Int. J. Adv. Res. Comput. Eng. Technol., vol. 3, no. 7, pp. 2274-2278, 2014.

[7] S. Yadav and S. S. Yadav, "Review for Leach Protocol in WSN," Int. J. Recent Dev. Eng. Technol., vol. 2, no. 6 , pp. 69-71, 2014.

[8] J. D. Gaurkar and K. Dhote, "Review paper on design of distributed Energy Efficient and Reliable Routing Protocol for Wireless Sensor Networks," Int. J. Res. Emerg. Sci. Technol., vol. 1, no. 4, pp. 41-46, 2014.

[9] R. U. Anitha and P. Kamalakkannan, "Enhanced cluster based routing protocol for mobile nodes in wireless sensor network," in 2013 International Conference on Pattern Recognition, Informatics and Mobile Engineering, 2013, pp. 187-193.

[10] R. U. Anitha, "EEDBC-M: Enhancement of LeachMobile protocol with Energy Efficient Density-based Clustering for Mobile Sensor Networks ( MSNs )," Int. J. Comput. Appl., vol. 74, no. 14, pp. 19-27, 2013.

[11] "The Network Simulator ns-2: Documentation." [Online]. Available: http://www.isi.edu/nsnam/ns/nsdocumentation.html. [Accessed: 09-May-2015]. 